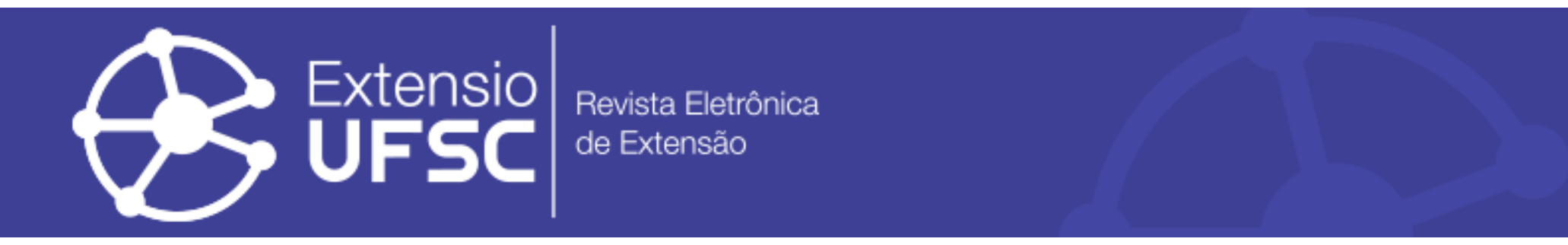

\title{
ATUAÇÃO FONOAUDIOLÓGICA NAS DISFUNÇÕES TEMPOROMANDIBULARES: UM RELATO DE EXPERIÊNCIA
}

\author{
Fernanda Berretta \\ Universidade Federal de Santa Catarina \\ feberretta@hotmail.com \\ Maynara Schlickmann Freitas \\ Universidade Federal de Santa Catarina \\ maynarafreitas@hotmail.com \\ Morgane Marion Kuntze \\ Universidade Federal de Santa Catarina \\ kuntze.morgane@gmail.com \\ Beatriz Dulcinéia Mendes de Souza \\ Universidade Federal de Santa Catarina \\ dentbia@gmail.com
}

André Luís Porporatti Universidade Federal de Santa Catarina andreporporatti@yahoo.com.br

Letícia Korb

Universidade Federal de Santa Catarina leti_korb@yahoo.com.br

Renata Coelho Scharlach Universidade Federal de Santa Catarina renata.scharlach@ufsc.br

Fabiane Stefani

Universidade Federal de Santa Catarina

fastefani@gmail.com

\begin{abstract}
Resumo
O objetivo deste trabalho foi relatar as atividades desenvolvidas no Projeto de extensão: Atuação Fonoaudiológica nas Disfunções Temporomandibulares da UFSC. Este projeto proporciona conhecimentos teóricos e práticos aos alunos de fonoaudiologia, permitindo que estes realizem o diagnóstico fonoaudiológico, planejamento e tratamento de pacientes com dor orofacial e DTM. Os alunos realizam avaliação fonoaudiológica específica para motricidade orofacial, com ênfase nas alterações relativas às DTMs, avaliações audiológicas e acompanham exames de eletromiografia de superfície. Planejam e executam a terapia fonoaudiológica, integrada ao tratamento odontológico. Essa abordagem visa a recuperação da função, o alívio da dor e a melhora da qualidade de vida destes pacientes. Os atendimentos oferecidos pelo projeto são de extrema importância, pois também complementam o tratamento realizado na Odontologia melhorando o prognóstico dos casos. O tratamento multidisciplinar contemplando diferentes áreas da saúde, proporciona o tratamento integral do paciente, devolvendo o seu bem-estar e qualidade de vida.

Palavras-chave: Disfunção Temporomandibular. Dor Orofacial. Fonoaudiologia. Odontologia.
\end{abstract}

\section{SPEECH AND HEARING THERAPY IN TEMPOROMANDIBULAR DISORDERS: A REPORT OF EXPERIENCE}

\begin{abstract}
The objective of this study is to report the activities carried out in the Extension Project: Speech and Hearing Therapy in Temporomandibular Disorders at UFSC. This project provides theoretical and practical knowledge to enable speech therapy students to perform diagnosis, planning and treatment of patients with orofacial pain and temporomandibular dysfunction. Students carry out specific speech-language assessments for orofacial motricity, with emphasis on the alterations related to temporomandibular disorders, perform audiological evaluations and use electromyography. They also plan and execute the treatment integrating Speech Therapy and Dentistry teams. This approach aims at recovery of function, relief of pain and improvement of the quality of life of these patients. These services offered by the project are extremely important, as they also complement the treatment performed in Dentistry. Moreover, they improve the prognosis of the cases. The multidisciplinary treatment treats the patient as a whole, and restores his well-being and quality of life.
\end{abstract}

Keywords: Temporomandibular Dysfunction. Orofacial Pain. Speech Therapy. Dentistry. 


\title{
ACTUACIÓN FONOAUDIOLÓGICA EN LAS DISFUNCIONES TEMPOROMANDIBULARES: UN INFORME DE EXPERIENCIA
}

\begin{abstract}
Resumen
El objetivo de este trabajo espresentear las actividades realizadas en el Proyecto de extensión: Actuación Fonoaudiológica en las Disfunciones Temporomandibulares de la UFSC. Este proyecto proporciona conocimientos teóricos y prácticos a los alumnos de fonoaudiología, permitiendo que éstos realicen el diagnóstico fonoaudiológico, planificación y tratamiento de pacientes con dolor orofacial y DTM. Los estudiantes realizan una evaluación fonoaudiológica específica para la motricidad orofacial, con énfasis en las alteraciones relativas a las DTM, evaluaciones audiológicas y acompañan exámenes de electromiografía. Planean y ejecutan la terapia fonoaudiológica, integrada al tratamiento odontológico. Este enfoque tiene como objetivo la recuperación de la función, el alivio del dolor y la mejora de la calidad de vida de estos pacientes. Las atenciones ofrecidas por el proyecto son de extrema importancia, pues también complementan el tratamiento realizado en la Odontología mejorando el pronóstico de los casos. El tratamiento multidisciplinario contemplando diferentes áreas de la salud, proporciona el tratamiento integral del paciente, devolviendo su bienestar y calidad de vida.

Palabras clave: Disfunción Temporomandibular. Dolor Orofacial. Terapia del Habla. Odontología.
\end{abstract}


Atuação fonoaudiológica nas disfunções temporomandibulares: um relato de experiência.

\section{INTRODUÇÃO}

A Disfunção Temporomandibular (DTM) é uma expressão coletiva que compreende uma variedade de problemas clínicos relacionados aos músculos mastigatórios, articulação temporomandibular (ATM) e estruturas associadas (LEEUW, 2010).

As DTMs apresentam etiologia multifatorial, geralmente associada a problemas anatômicos, traumas, inflamações ou degenerações da ATM, fatores psicossociais e hábitos deletérios (KRISHNAMOORTHY et al, 2013). Os hábitos deletérios mais discutidos na literatura e que prejudicam os indivíduos propensos a desenvolver uma DTM são bruxismo (apertar ou ranger os dentes durante o sono), roer unhas, mastigação unilateral, mastigação de chicletes, má-postura corporal, dormir com as mãos sob a cabeça, morder o lábio, as bochechas ou outros objetos (ZANINI, 1999). Segundo Okeson (2000), alguns fatores etiológicos, incluindo distúrbios de sono, stress físico e emocional podem diminuir a capacidade adaptativa do sistema estomatognático e levar à ocorrência da doença.

As DTMs podem ser subdivididas em desordens musculares e articulares (LEEUW, 2010). As DTMs musculares se apresentam como mialgia local, dor miofascial, mialgia mediada centralmente, mioespasmos, contratura miofribrótica e neoplasias. As articulares são subdivididas em desordens do desarranjo do disco (deslocamento do disco com ou sem redução), deslocamentos da ATM, desordens inflamatórias (sinovite e capsulite, osteoartrite), desordens não inflamatórias (osteoartrose), anquilose e fratura do processo condilar (LEEUW, 2010).

Os sinais mais encontrados ao avaliar um paciente são sensibilidade à palpação na musculatura orofacial e na ATM, limitação dos movimentos mandibulares e ruídos articulares (LIST \& JENSEN, 2017). Já os sintomas mais referidos são dores orofaciais, ou seja, dores na face, na cabeça, nas ATMs, e/ou nos músculos mastigatórios, (CARRARA et al., 2010). Dentre as dores que acometem a região orofacial, a DTM é a segunda queixa mais comum nos consultórios odontológicos, depois somente das dores dentárias (FERNANDES et al., 2007).

Estudos epidemiológicos indicam que aproximadamente $10 \%$ a $15 \%$ da população apresenta o diagnóstico de DTM (KUMANOVICK PFICER et al., 2017). No entanto, alguns autores relatam que um número bem maior relata ao menos algum sinal ou sintoma desta doença (CARRARA et al., 2010; LEEUW, 2010; SRIVASTAVA, JYOTI \& DEVI, 2013). A DTM tem sua maior prevalência em indivíduos entre 20 e 45 anos de idade (BIASOTTO-GONZALEZ, 2005; SRIVASTAVA et al. 2013; KUMANOVICK PFICER et al., 2017), sendo que até os 40 anos, o principal fator etiológico é de origem muscular, e acima desta idade, é a degeneração articular (BIASOTTOO-GONZALEZ, 2005). Há um consenso na literatura de que indivíduos do 
Atuação fonoaudiológica nas disfunções temporomandibulares: um relato de experiência.

sexo feminino apresentam maior prevalência de DTM (BARBOSA \& ALVES, 2010; FERREIRA et al., 2016; KUMANOVICK PFICER et al., 2017). Fatores emocionais, anatômicos, mudanças hormonais associadas à menstruação, frouxidão ligamentar, além da maior demanda das mulheres na procura por tratamento justificam esta alta prevalência (BARBOSA \& ALVES, 2010).

O tratamento das DTMs é multidisciplinar e inúmeras terapias podem ser implementadas, podendo englobar medicações, agulhamentos e/ou infiltrações, dispositivos interoclusais, fisioterapias, terapia cognitivo-comportamental e terapia fonoaudiológica (GONZALEZ-PEREZ et al., 2012; LIST \& JENSEN, 2017). A Fonoaudiologia é a ciência que se preocupa com a comunicação humana. Para tanto, estuda o sistema estomatognático e suas funções (sucção, respiração, mastigação, deglutição e fala). Um dos objetivos da terapia fonoaudiológica é complementar outros tratamentos das DTMs, em especial o odontológico. A terapia fonoaudiológica busca adequar os músculos e a movimentação da mandíbula durante a fala, mastigação, deglutição e postura habitual da boca e mandíbula, atuando no equilíbrio dos grupos musculares que auxiliam nas funções orais. A terapia objetiva, da mesma forma, a redução da dor e mudanças dos hábitos deletérios, como apertar os dentes, roer unhas, morder objetos, pressionar a língua contra os dentes, entre outros (STEFANI, 2013).

A severidade da dor está relacionada às atividades dos músculos mastigatórios e, portanto, a dor afeta diretamente as funções que este grupo muscular realiza, tais como a mastigação e a fala. Assim, a promoção de relaxamento da musculatura facial e cervical, bem como a melhora circulatória por meio de massagens e alongamentos são indicados como tratamento. Essas terapias físicas manuais podem reduzir a dor e auxiliar no restabelecimento da função muscular (STEFANI, 2013).

A maioria dos sintomas de DTM afetam sobremaneira a qualidade de vida do paciente com alto impacto na saúde. Desta forma, com a intenção de fornecer suporte a estes pacientes na região de Florianópolis, os professores do curso de Fonoaudiologia da Universidade Federal de Santa Catarina (UFSC) elaboraram este projeto de extensão. O propósito deste trabalho foi relatar as atividades e os tratamentos realizados pelo projeto de extensão: Atuação Fonoaudiológica nas Disfunções Temporomandibulares.

\section{MATERIAIS E MÉTODOS}

O projeto de extensão da Fonoaudiologia atua em parceria com o Centro Multidisciplinar de Dor Orofacial (CEMDOR) da UFSC realizando diagnóstico, tratamento e acompanhamento 
Atuação fonoaudiológica nas disfunções temporomandibulares: um relato de experiência.

de pacientes com DTM, incluindo disfunções musculares e articulares. Foi criado em 2015 pela professora Fabiane Miron Stefani do departamento de Fonoaudiologia da UFSC em parceria com a professora Graziela de Luca Canto do departamento da Odontologia e a partir de 2016, este projeto se vinculou ao CEMDOR coordenado pelos professores André Porporatti e Beatriz D. M. Souza, do departamento de Odontologia da UFSC. Este projeto visa oferecer um trabalho multidisciplinar e também complementar o tratamento realizado no CEMDOR. A cada semestre, cerca de 20 pacientes são atendidos na clínica de fonoaudiologia por alunos de quinta à oitava fases do Curso de Fonoaudiologia. Os atendimentos acontecem semanalmente às quintas feiras no período da manhã propondo a recuperação da função, o alívio da dor e a melhora da qualidade de vida desses pacientes.

Muitos pacientes são encaminhados pelo CEMDOR para avaliação fonoaudiológica e respectivo tratamento. Após anamnese e exame clínico, os tratamentos são implementados. Outros pacientes chegam através do curso de Fonoaudiologia e após o diagnóstico fonoaudiológico, são encaminhados ao CEMDOR para o tratamento odontológico, que pode abordar prescrição farmacológica, confecção de placas oclusais, homeopatia, agulhamento seco ou com anestésico, acupuntura e viscosuplementação da articulação temporomandibular (ATM) com o hialuronato de sódio.

O principal objetivo no tratamento da DTM é reduzir ou cessar a dor, recuperar a função do aparelho estomatognático e amenizar os fatores que mantém o problema (CARRARA et al., 2010). Diferentes terapias fonoaudiológicas podem ser implementadas aos pacientes. Estes recebem terapias miofuncionais orofaciais (Figura 1), orientações sobre alimentação, respiração, sono e deglutição.

A terapia fonoaudiológica tem como objetivo melhorar a tonicidade e mobilidade da musculatura mastigatória e facial, bem como as funções de mastigação, deglutição, respiração e fala. Organiza-se a terapia fonoaudiológica na seguinte sequência, também preconizada na literatura (STEFANI, 2013):

Orientações/retirada de hábitos;

\section{Termoterapia;}

Massagens;

Relaxamento da musculatura cervical; 
Atuação fonoaudiológica nas disfunções temporomandibulares: um relato de experiência.

\section{Terapia Miofuncional Orofacial;}

Trabalho específico das ATMs;

Terapia das funções estomatognáticas.

Essas terapias desenvolvidas para o tratamento dependem principalmente do aceite e colaboração do paciente para que todas as etapas acima descritas sejam executadas corretamente. Cada etapa da terapia desenvolve uma função que permite o sucesso do tratamento fonoaudiológico.

As orientações ao paciente se permeiam em torno dos procedimentos terapêuticos aplicados e relacionados aos maus hábitos que prejudicam e levam a dor orofacial. Assim, o trabalho conjunto profissional/paciente deve ser realizado para que os maus hábitos sejam reduzidos e/ou removidos.

A termoterapia, neste caso, se restringe ao calor úmido. É uma etapa importante que visa a melhora do metabolismo tecidual da área mediando o processo inflamatório na região e contribuindo no alívio da dor por meio da vasodilatação e aumento do fluxo sanguíneo (GUTMANN, 1980). A orientação ao paciente é que aplique o calor úmido $\left(45^{\circ} \mathrm{C}\right)$ por 25 minutos nos músculos faciais

Logo após a aplicação de calor úmido, podem ser realizadas massagens na musculatura facial e cervical. Estas podem ser circulares, de alongamento muscular (no sentido das fibras musculares) ou com pequenos toques rápidos com as pontas dos dedos. Além dessas massagens, na região de ombros podemos lançar mão também do amassamento feito com as palmas das mãos.

Os exercícios miofuncionais orofaciais são semelhantes àqueles preconizados normalmente a pacientes com alterações do sistema miofuncional orofacial, levando-se em conta algumas peculiaridades dos pacientes com DTM, como a dor. Outra característica destes pacientes é a presença de estalos nas ATMs, que podem afetar a qualidade de vida dos indivíduos e vir acompanhados de desvios na trajetória da mandíbula.

Posteriormente são realizados exercícios específicos para a movimentação mandibular e, por fim, exercícios para adequação das funções estomatognáticas: mastigação, respiração, deglutição e fala (STEFANI, 2013). 


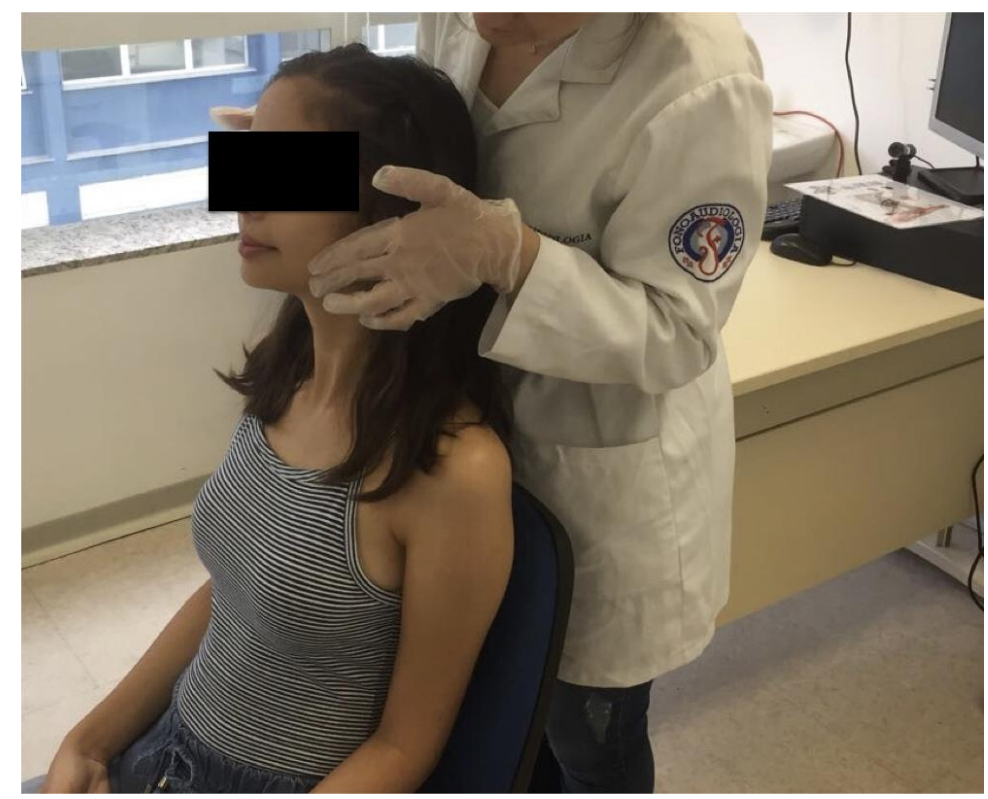

Figura 1- Terapias miofuncionais orofaciais

\section{RESULTADOS E ANÁLISES}

No atendimento especializado e multidisciplinar de pacientes com DTM, o projeto de extensão da Fonoaudiologia em parceria com o CEMDOR favorece a remissão dos sinais e sintomas desta disfunção, proporcionando melhor qualidade de vida a estes indivíduos.

A extensão oferecida aos alunos permite o aprendizado teórico e prático em um campo em ascensão na odontologia e fonoaudiologia, incentivando e salientando a importância do trabalho em equipe com outras especialidades. Assim, este projeto oportuniza um espaço de aprendizado aos alunos da Fonoaudiologia, com visão multidisciplinar integrada, por meio da atuação conjunta e as supervisões multiprofissionais.

Neste programa existem diversas linhas de pesquisa sendo desenvolvidas com base nos atendimentos a esses pacientes, com apoio de equipamentos sofisticados como eletromiografia de superfície (figura 2), e inovadora, como as técnicas de uso de bandagem elástica terapêutica, e de forma interdisciplinar, associadas a exames audiológicos e de vestibulometria (figura 3). A eletromiografia de superfície (EMG), é um método não invasivo de avaliação muscular que é comumente utilizada para mensuração dos potenciais elétricos dos músculos da mastigação (RODRIGUES et al., 2015). A aplicação da bandagem elástica é uma alternativa de terapia terapêutica muscular, que tem como propósito a estimulação das vias nervosas aferentes e eferentes, adicionando sensibilidade e percepção aos estímulos captados. Desta forma, o seu uso pode contribuir para a diminuição da pressão exercida nos receptores sensoriais, e quando associada à terapia fonoaudiológica tradicional pode promover a diminuição do quadro álgico em 
Atuação fonoaudiológica nas disfunções temporomandibulares: um relato de experiência.

um número menor de sessões quando relacionada à terapia tradicional isolada (HERNANDES et al., 2017). Os exames audiológicos englobam a anamnese, audiometria tonal liminar, logoaudiometria, imitanciometria e emissões otoacústicas evocadas. Além disso, os pacientes com zumbido, respondem à versão brasileira do questionário Tinnitus Handicap Inventory (THI) (DIAS et al., 2006) e realizam a acufenometria. A medida da acufenometria é realizada a fim de se obter o pitch e loudness do zumbido para cada orelha separadamente (BRANCO-BARREIRO, 2004). A avaliação vestibular contempla um conjunto de procedimentos para avaliar o sistema vestibular e suas conexões com os sistemas ocular e proprioceptivo, cerebelo, medula espinhal e a formação reticular no tronco encefálico (Mor \& Fragoso, 2012). Os pacientes com queixas vestibulares são, inicialmente, encaminhados para avaliação médica e, se necessário, realizam as avaliações específicas.

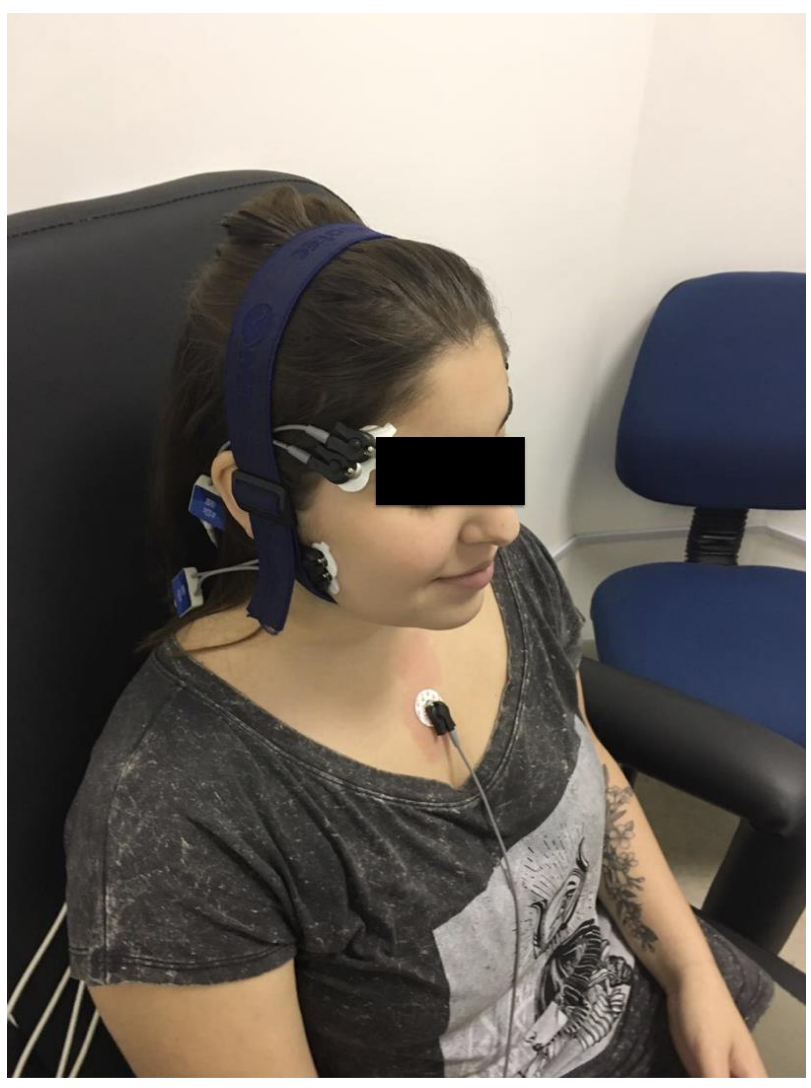

Figura 2 - Eletromiografia de superfície 


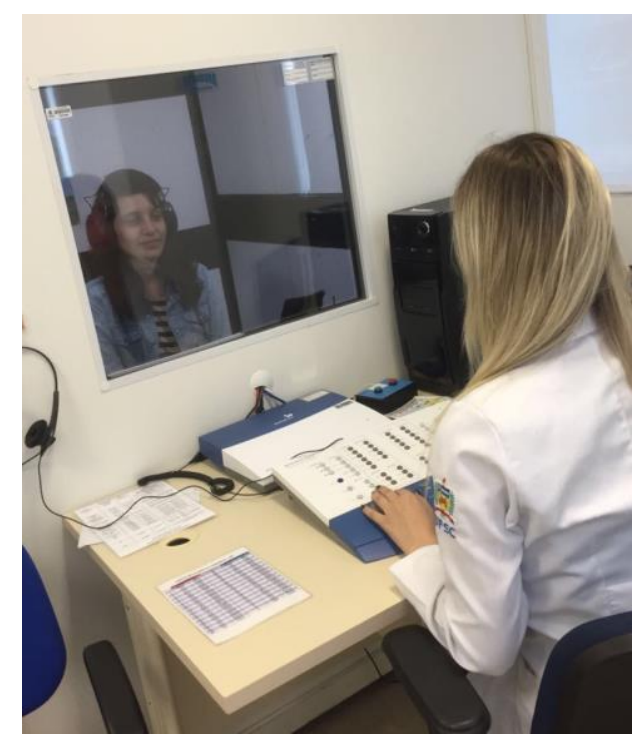

Figura 3 - Exames audiológicos

\section{CONSIDERAÇÕES FINAIS}

Com o crescimento da demanda de pacientes com DTMs e dores orofaciais nas clínicas de saúde, surgiu a necessidade da criação de projetos para suprir este público. Porém, em virtude de sua natureza multifatorial, é indispensável a criação de equipes multidisciplinares para que pudessem estabelecer um correto diagnóstico e um completo e adequado tratamento desta enfermidade.

Desta forma, a integração de áreas como a fonoaudiologia e a odontologia, assim como das outras áreas envolvidas, permite o principal objetivo do tratamento, o bem-estar e a melhora na qualidade de vida dos pacientes e proporciona aos alunos importante vivência clínica.

\section{REFERÊNCIAS}

LEEUW, R. Dor orofacial: guia de avaliação, diagnóstico e tratamento. Quintessence, São Paulo, $4^{\mathrm{a}}$ ed., 2010.

KUMONOVICK PFICER, J. et al. Occlusal stabilization splint for patients with temporomandibular disorders: meta-analysis of short and long term effects. Plos One, v.12, n.2, p.1-21, 2017.

CARRARA, S.V; CONTI, P.C.R.; BARBOSA, J.S. Termo do $1^{\circ}$ Consenso em Disfunção Temporomandibular e Dor Orofacial. Dental Press J Orthod, São Paulo, v. 3, n. 15, p.114-120, 2010.

SRIVASTAVA, R; JYOTI, B.; DEVI, P. Oral splint for temporomandibular joint disorders with revolutionary fluid system. Dent Res J, v. 10, n.3, p. 307-313, 2013. 
Atuação fonoaudiológica nas disfunções temporomandibulares: um relato de experiência.

BIASOTTO-GONZALEZ, D.A. Abordagem interdisciplinar das disfunções temporomandibulares. 7. ed. São Paulo: Manole. 2005.

BARbosa, G. A.; ALVES, A. C. M. CIADE - Centro Integrado de Atendimento a Portadores de Disfunção do Aparelho Estomatognático. Rio Grande do Norte: Proex, 2010.

FERREIRA, C.L.P.; SILVA, M.A.M.R.; FELÍCIO, C.M. Signs and symptoms of temporomandibular disorders in women and men. Rev. Codas, v. 28, n. 1, p. 17-21, 2016.

KRISHNAMOORTHY, B.; MAMATHA, N.S.; KUMAR, V.A. TMJ imaging by CBCT: Current scenario. Ann Maxillofac Surg, v. 3, p. 80-3, 2013.

ZANINI, C.F.C. Os hábitos parafuncionais na disfunção da articulação têmporomandibular. 1999. Monografia (Especialização em Fonoaudiologia) - CEFAC, Porto Alegre.

1OKESON, J.P. Tratamento das desordens temporomandibular e oclusão. $4^{a}$ ed. São Paulo: Editora Artes Médicas, 2000. 500p.

FERNANDES, A.U.R. et al. Desordem temporomandibular e ansiedade em graduandos de odontologia. Cienc. Odontol. Bras, v.10, n.1, p. 70-77, 2007.

GONZALEZ-PEREZ, L.M. et al. Treatment of temporomandibular myofascial pain with deep dry needling. Med Oral Patol Oral Cir Bucal, s.l., p.781-785, 2012.

GUTMANN L. Heat-induced myasthenic crisis. Arch Neurol. Oct;37(10):671-2, 1980.

LIST, T.; JENSEN, R. H. Temporomandibular disorders: Old ideas and new concepts. Cephalalgia, Sweden, v. 13, n. 1, p.1-13, jan. 2017.

STEFANI, S.M. Intervenção fonoaudiológica nas disfunções temporomandibulares. In: Novo tratado de fonoaudiologia/editor Otacílio Lopes Filho, et al. $3^{\mathrm{a}}$ ed. Barueri, SP: Manole, 2013.

RODRIGUES, C.A.; MELCHIOR, M.O.; MAGRI, L.V.; MESTRINER, Jr W.; MAZZETTTO, M.O. Is the Masticatory Function Changed in Patients with Temporomandibular Disorder. Braz. Dent. J, v. 26, n. 2, p. 181-5, 2015. Disponível em: http://dx.doi.org/10.1590/0103$\underline{6440201300198}$

HERNANDES, N.C.J. et al. Atuação fonoaudiológica em disfunção temporomandibular em dois casos: análise comparativa dos efeitos da terapia tradicional e o uso da bandagem terapêutica associada. Distúrbios da Comunicação, v. 2, n. 29, p. 251-261, Jun 2017.

DIAS, A.; CORDEIRO, R.; CORRENTE, J. Incômodo causado pelo zumbido medido pelo Questionário de Gravidade do Zumbido. Rev de Saúde Pública. v. 40, n. 4, p. 706-11, 2006. Disponível em: http://www.scielosp.org/pdf/rsp/nahead/ao-5028.pdf

BRANCO-BARREIRO, F.C.A. Avaliação audiológica básica e psicoacústica do zumbido. In: Samelli A.G. Zumbido: avaliação, diagnóstico e reabilitação - Abordagens atuais. São Paulo: Lovise; 2004. 55-59. 
Atuação fonoaudiológica nas disfunções temporomandibulares: um relato de experiência.

MOR, R.; FRAGOSO, M. Vestibulometria na Prática Fonoaudiológica. São Paulo: Pulso Editorial; 2012. 